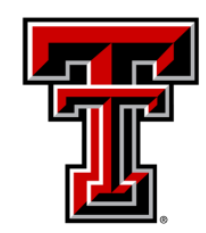

TEXAS TECH UNIVERSITY

Libraries"

\title{
REFERENCE IN 160 ChARACTERS OR LeSS: THE ROLE OF TeXT MesSaging in ViRTUAL Reference Services
}

\section{The Texas Tech community has made this publication openly available. Please share how this access benefits you. Your story matters to us.}

\begin{tabular}{|l|l|}
\hline Citation & $\begin{array}{l}\text { Vardeman, K. \& Barba, I. (2014). Reference in 160 characters or } \\
\text { less: The role of text messaging in virtual reference services. } \\
\text { Internet Reference Services Quarterly, 19(3-4), 163-179. } \\
\text { https://doi.org/10.1080/10875301.2014.984098 }\end{array}$ \\
\hline Citable Link & $\underline{\text { http://hdl.handle.net/2346/68141 }}$ \\
\hline Terms of Use & $\underline{\text { CC-BY }}$ \\
\hline
\end{tabular}


Reference in 160 Characters or Less: The Role of Text Messaging in Virtual Reference Services KIMBERLY K. VARDEMAN and IAN BARBA

University Libraries, Texas Tech University, Lubbock, Texas, USA

\begin{abstract}
This study focuses on how and why academic library patrons choose text message reference, particularly when other mediums are available. Researchers evaluated three years of text reference data to assess the peak usage times, types of questions received, the number of unique and repeat users, and the complexity of questions. Our analysis of data shows that text reference can fill a role within virtual reference services. We further identify reasons why text message services have not seen greater use, including students' lack of awareness, limitations of the medium, and patron satisfaction with other modes of reference.
\end{abstract}

Keywords: Virtual reference, SMS reference, Text reference, Mobile devices 


\section{Reference in 160 Characters or Less:}

The Role of Text Messaging in Virtual Reference Services

In May 2011, Texas Tech University Libraries added text message reference to its suite of virtual reference services. Through Mosio's Text a Librarian service (now known as Mosio for Libraries), reference librarians are able to receive and respond to text messages from patrons via an online interface. The Libraries had been interested in experimenting with text reference as it grew more popular with academic libraries following Sims Memorial Library's experiment with text reference in 2005 (Hill, Hill, \& Sherman, 2007). TTU librarians selected Mosio's subscription service for a number of reasons: ease of use for patrons, ease of integrating with existing reference services, affordability, and patron privacy.

Our study aims to fill a gap in the literature by analyzing three years of data-a more longitudinal approach than most previously conducted studies. Unlike studies measuring user preference and librarian competencies necessary for text reference, we focus on usage data. We further include discussion of the nature of questions received, comparison with other virtual reference mediums, as well as an examination of how patrons' use of the service evolved over time. By analyzing this data, we seek to answer questions about how, when, and why academic library patrons choose to use text reference, and whether it allows us to reach patrons we were previously unable to help. In doing so, we hope to inform librarians about the strengths and limitations of text reference as well as the role it plays in virtual reference services.

\section{Literature Review}

At first, libraries were clearly taking baby steps regarding this new (to them) technology and the literature reflects that. For example, Cole and Krkoska (2011) began a text reference service at Cornell University Library in 2009, improving the technology and workflows in tandem with testing. Brooks and Zubarev (2012) noted that their text reference experiment involved passing around a single BlackBerry, rather than integrating the service with a web interface like Mosio. Much of the literature revolves around a particular library's first experiences with the medium, essentially case studies regarding text reference. The adoption of text reference powered by Altarama's "Reference by SMS" system at Southeastern Louisiana University's Sims Memorial Library appears to be the first published account (Hill et al., 2007). Librarians at Sims Memorial Library were interested in whether or not the nature of the medium would have any effect upon the types of questions asked, and they received a majority of short-answer 
questions. Literature by early adopters of text reference is quite similar. Libraries typically cited the prevalence of the technology as impetus for experimentation (Herman, 2007), and illustrate their experiences with text reference-almost like a "how to." Breitbach and Prieto (2012) found that while their student body overwhelmingly reported high usage of and familiarity with SMS, the students typically did not use text reference as a service.

Quite often, the time periods covered by these studies were short, generally less than a year. Frequently, as Cole and Krkoska (2011) of Cornell University Library related, their initial experience with text reference guided development of the service, in their case through development of best practices. Insufficient promotion and low student awareness of the service are often barriers to success. Indeed, Brooks and Zubarev (2012) of Lloyd Sealy Library at John Jay College of Criminal Justice, City University of New York found that when they surveyed students, students were unaware of the existence of text reference. Further, the surveys used to collect data about their service may have resulted in an awareness bump.

Since those early case studies, libraries have moved into assessment of text reference. Some libraries measure patron preference for text reference by comparing it to other reference mediums. Chow and Croxton (2012) found that faculty, staff, and students tended to prefer face-to-face reference over virtual reference. In their later study specifically on virtual reference mediums, they found that surveyed patrons preferred chat and email to text reference, with chat scoring significantly higher in both effectiveness and efficiency (Chow \& Croxton, 2014). However, in another study, Ruppel and Vecchione (2012) of Boise State University measured satisfaction of text, chat, and face-to-face separately; they found that all three mediums scored very highly in user satisfaction. As opposed to measuring patron satisfaction with the medium, other case studies focused on assessing the nature of questions posed by patrons. In a study by librarians at New York University Libraries, librarians analyzed text reference data by question type, length, time, location, and time to librarian response (Pearce, Collard, \& Whatley, 2010). By assessing text reference in this way, they were able to collect different information about how patrons actually use the medium.

In their 2012 article, Chow \& Croxton discuss the applicability of Poole's Principle of Least Effort to information-seeking behavior. This principle suggests that information seekers generally attempt to 
engage in sufficient effort to meet their needs and nothing further (Poole, 1985), which may also explain the difference Chow \& Croxton (2012) found in preference for reference mediums between faculty, graduates, and undergraduates. In their survey, faculty and graduate students generally did not prefer text reference, whereas undergraduates showed a higher preference for text messaging. As Hill et al. (2007) found, it is possible patrons view text reference as the "least effort" option for short or simple questions, and view the service as less capable for longer or more complex questions.

Instead of focusing on the patron side of text reference, other literature focused on the librarian. Specifically, these studies measured the skills necessary for librarians to engage in effective text reference as a service. Cassidy, Colmenares, and Martinez (2014) noted that librarians used different skills during text reference (as opposed to other mediums) and proper training is necessary in order to deliver effective and efficient text reference. Because of the limitations of SMS service (160 characters), efficiency is perhaps even more important than it is in other reference mediums. Lili Luo (2012), of San Jose State University, found that librarian competencies necessary for text reference were similar to other mediums, including chat and face-to-face, though the competencies differed in order of importance. Sims Memorial Library reached a similar conclusion in a follow-up study five years after their initial experience (Stahr, 2010). They found that the necessary skills for text reference were sufficiently similar to other mediums and that academic libraries would be wise to experiment with text reference. Experience appears the best way to become fluent in what Stahr (2010) termed "textese," which is the primary difference between text reference and other mediums.

Though the literature suggests text reference as a service has matured beyond early experimentation, by no means has the subject been thoroughly covered. There are holes in the literature, particularly regarding the comprehensiveness of data kept. Most of the studies with a focus on usage data covered a relatively short time span. Brooks and Zubarev (2012) covered ten months, Cole and Krkoska (2011) a little over two months, and Pearce et al. (2010) one year. Other studies of text reference focus on survey data. Chow and Croxton (2014) and Ruppel and Vecchione (2012), despite surveying similar populations, found different levels of user satisfaction with text reference. This difference may have resulted from nothing more than a difference in the way they worded their survey questions. While survey data is useful, we feel it is no substitute for actual usage data. 


\section{Background}

Texas Tech University is a large public university in Lubbock, Texas, serving approximately thirtythree thousand students (Fall 2013 enrollment), with a goal of expanding enrollment to forty thousand by 2020. As a member of the Association of Research Libraries, the Texas Tech University Libraries provide support for teaching and research in the ten academic colleges on campus. While there are three libraries on campus, the University Library is the main library serving students and faculty across all disciplines with the exception of the College of Architecture. The two other libraries are specialized: the Southwest Collection/Special Collections Library (archive) and the Architecture Library. In order to effectively deliver reference services, reference librarians have subject specialist fields and liaison responsibilities with students and faculty in their departments regarding instruction, outreach, and other services.

In addition, the University Library employs two general reference librarians who provide research assistance at the primary service desk, a merged reference/circulation desk located near the main entrance of the University Library. A general reference librarian works at the main Service Desk from 9:00 a.m. - 5:00 p.m., Monday-Thursday, and 9:00 a.m. - 2:00 p.m. on Friday. Occasionally, subject specialist reference librarians will substitute at the desk, but the bulk of the hours are the responsibility of the general reference librarians. The Service Desk is also staffed by student assistants and circulation staff whose duties include item circulation, security, course reserve circulation, and basic reference assistance such as "known item" searching. Circulation staff and student assistants further operate the two other service desks without reference librarians. 


\section{Where can I find theatre history books?}

[TextSpeak Translator]

patron878731 (2 Q)

Mon Mar 10, 2014 11:33am

\section{answer}

- OR -

Close this question

Click "close" to mark this

question as answered.


\section{Close this question}

If you are finished with this question (no follow-up needed), click Close this question

Permalink

\section{FIGURE 1 Mosio's Text a Librarian Microboard}

The subject specialists and general reference librarians are typically available for face-to-face instruction or consultation during standard 9:00 a.m. to 5:00 p.m. business hours during the week, with chat reference services also provided during those hours. Email services and appointments by request allow the Libraries to extend service to patrons outside these hours. In May 2011, the Libraries added text reference, using Mosio's Text a Librarian service. This gave patrons another way of consulting librarians during or outside regular business hours.

This service was an attractive choice in part for its web interface; rather than playing relay with a mobile phone, librarians answer all text message questions via an online interface, with no special software required to manage the service. Comments or questions from patrons appear in a queue on the "microboard," Mosio's term for their web interface (Figure 1). When new questions arrive, an alert sounds and a notification appears in the microboard, and librarians can elect to receive notifications of incoming 
texts via instant message, email, or text message. Librarians can then type responses to patron questions from the microboard. Text messages have a 160 character limit, meaning longer answers will send multiple text messages to patrons. Librarians may convert web links included in answers to shortened bit.ly links. A librarian username—for example, "KimberlyV"—appears by default on the response sent to the patron, who receives the response via text message within seconds.

For library patrons, the necessary routing of questions and responses through the microboard is invisible, with librarians' replies appearing on their phones much like any text message they would receive from friends and family. Patrons text questions to 66746 (mosio), starting their first text with the library keyword set by the library, e.g., ttulib. Upon sending their first text, patrons automatically receive the message: "We received your question. Please save this number in your phone." If patrons forget to begin the first text question with the library keyword, they receive an automated response that directs them to include the keyword or contact the library to obtain it. After sending their first text, patrons do not have to start their text message with the keyword, and are able to send texts like they would to any other number.

\section{Research Questions}

This study sought to answer several research questions through the analysis of usage data:

- How and when will patrons use the service most?

- Does the service have many or few unique users?

- Do repeat users ask progressively more complicated questions?

- Is the service reaching a new user population?

- What role is text reference filling?

\section{Methodology}

We exported full transcripts of all text reference service transactions from implementation in May 2011 through July 2014. The data from Mosio's microboard included a unique Question ID, a Patron ID number, the date and time of incoming questions, the text of questions received, as well as the date, time, and text of outgoing responses, the username of the librarian responder, and time elapsed (seconds) to first librarian response.

Following the strategy employed by Cassidy et al. (2014), the researchers grouped all incoming and outgoing text messages into transactions. Each transaction consisted of all incoming and outgoing 
messages with a particular patron during a relatively short amount of time, constituting a single session. If the same patron asked a question hours or days later, we counted that as a new transaction.

Researchers assessed all transactions and categorized each with one of the following descriptors:

\begin{tabular}{|l|l|l|}
\hline \multicolumn{3}{|c|}{$\begin{array}{c}\text { TABLE 1 } \\
\text { Descriptive Category for Questions Received (N=516) }\end{array}$} \\
\hline Category & $n$ & $\%$ of Transactions \\
\hline Reference & 192 & $37.2 \%$ \\
\hline Directional & 87 & $16.9 \%$ \\
\hline Equipment & 74 & $14.3 \%$ \\
\hline Circulation & 52 & $10.1 \%$ \\
\hline Hours & 30 & $5.8 \%$ \\
\hline Other & 27 & $5.2 \%$ \\
\hline Test & 20 & $3.9 \%$ \\
\hline Electronic Resources & 19 & $3.7 \%$ \\
\hline Complaints/Suggestions & 11 & $2.1 \%$ \\
\hline Interlibrary Loan & 4 & $0.8 \%$ \\
\hline
\end{tabular}

- Reference - requires the use of library catalog or resources to answer question. Ex: "Where can I find Emma by Jane Austen?" or "Where can I find empirical articles over adolescent psychology?"

- Directional - any question that does not require knowledge or use of library systems (catalog, etc.). Ex: "Where is the Digital Media Studio?"

- Equipment - questions or problems related to library equipment. Ex: "Why isn't my paper printing?"

- Circulation - questions about Circulation policies, patron accounts, and room reservation requests. Ex: "Can you renew my books?"

- Interlibrary Loan - questions related to interlibrary loan or the ILLiad online request software. Ex: "I can't log into ILLiad because I'm disavowed. Why?"

- Electronic Resources. Ex: "I am unable to connect to the EBSCO databases."

- Hours. Ex: "When does the library close?"

- Complaints/Suggestions. Ex: "The library is too cold."

- Test - questions or comments expressly about trying out the service or including the word "Test." 
- $\quad$ Other - nonsensical questions. Ex: "How many licks does it take to get to the center of a tootsie pop?

Researchers additionally tagged each transaction that included a thank you or compliment of the service.

\section{Findings}

The exported data consisted of a total of 852 incoming text messages and 695 outgoing responses. These 1,547 messages (incoming and outgoing) were grouped into 516 transactions. We categorized a plurality of questions received via the text service as reference questions. However, almost half of the questions were simpler in nature, including directional, equipment, and circulation questions, or questions about library operating hours-all questions that paraprofessional staff could answer. See Table 1 for the number and percentage of texts in each category. The high number of texts expressing gratitude for services rendered was noteworthy. Of the 852 messages received, 127 included a "thank you" or similar sentiment.

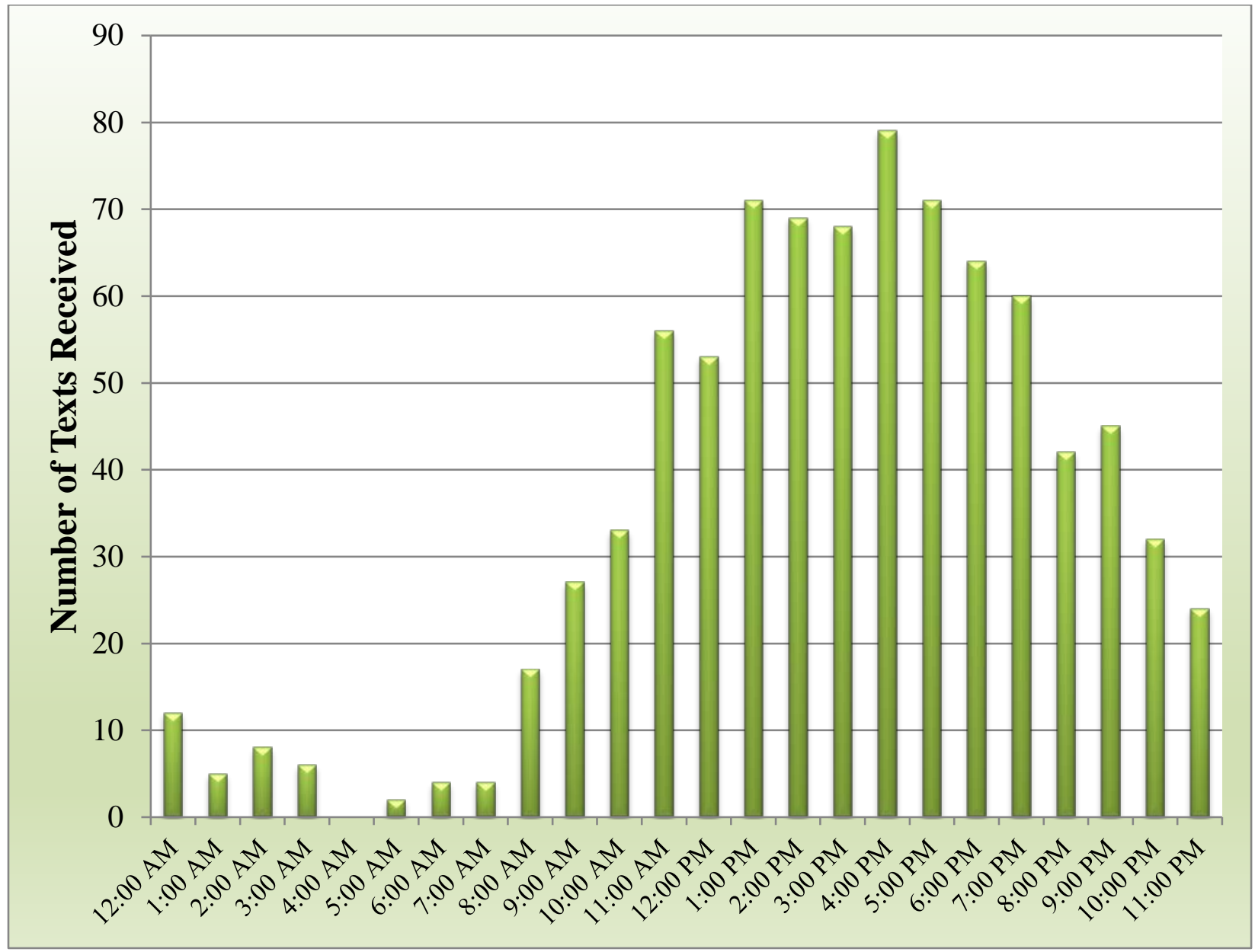

FIGURE 2 Number of Texts Received by Hour of Day 
The hours of the day that patrons sent the most questions were between 1:00 and 6:00 pm. The 4:00 p.m. hour received the most texts, and the 4:00 a.m. hour received the fewest. The 9:00 and 10:00 a.m. hours garnered fewer texts than any hour between 11:00 a.m. and 9:00 p.m. (Figure 2).

Wednesdays received more texts than any other day of the week, though weekdays were all close. Sundays received fewer texts, and Saturdays received the fewest number of texts by far, less than half the total received on Wednesdays (Figure 3).



FIGURE 3 Day of Week Questions Received

The exported data included the time elapsed in seconds between the first incoming text in a transaction and the librarian's response. There were three transactions in which a librarian never responded (as the questions were test or nonsense questions not requiring a response), and these were excluded from the total of 516 transactions. The response time was generally quick, with a librarian 
responding to about two-thirds of questions within 15 minutes. Longer response times corresponded to questions that arrived between 10 p.m. -5 a.m. or during weekends (Figure 4).

- Median response time was 7.1 minutes (430 seconds)

- 297 questions (57.8\%) had a response time of less than 10 minutes

- 424 questions (82.7\%) had a response time of less than 1 hour

- 89 questions (17.3\%) had a response time of more than 1 hour

- 4 questions $(<1 \%)$ had a response time of more than 24 hours

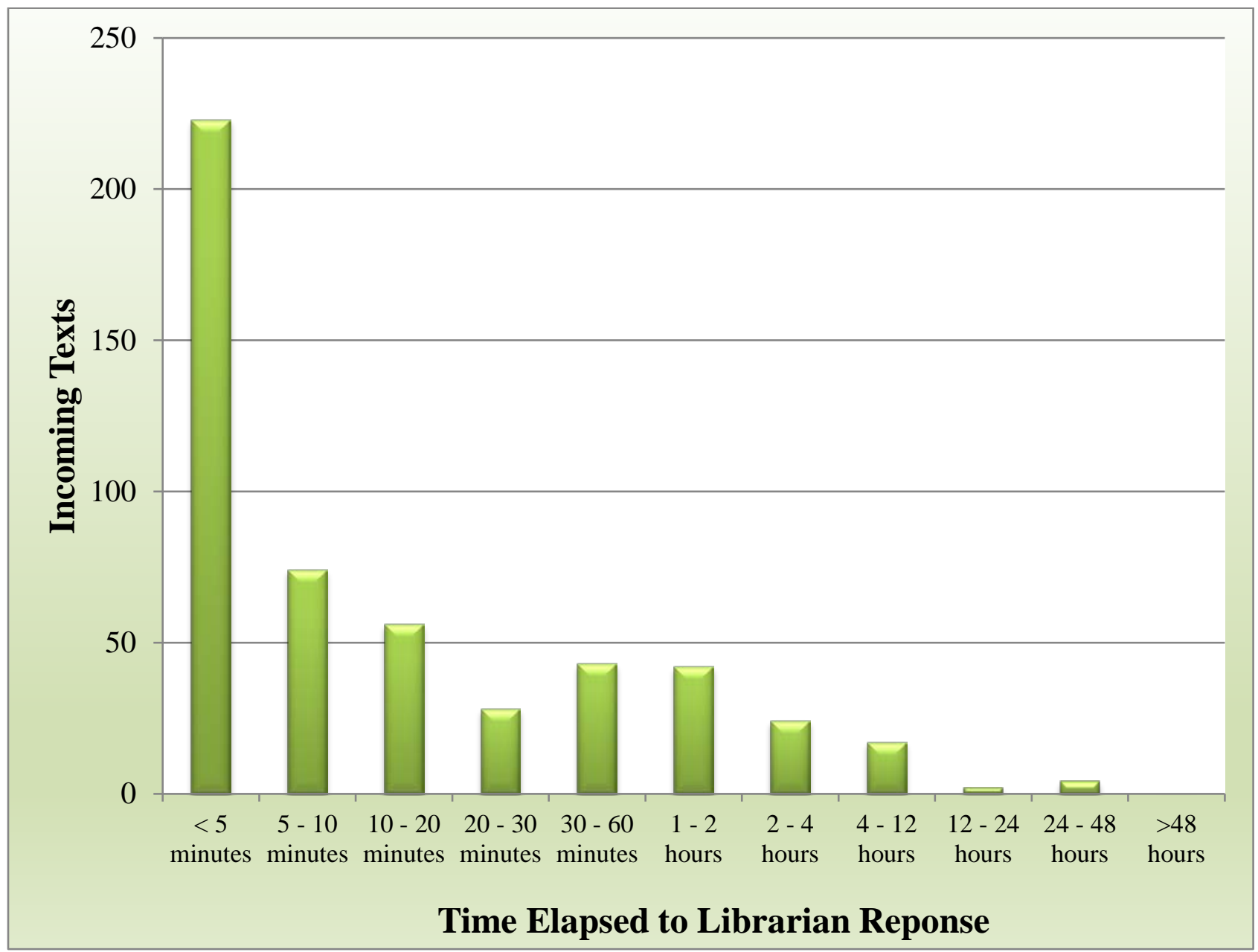

FIGURE 4 Librarian Response Time

As the exported data did not include a character count for messages, we copied the complete text of each incoming and outgoing message and pasted the text into an online character counter. Where questions from a patron arrived in multiple text messages_-for example, if the character count exceeded 160-the character count for each individual text message was added together. Thus, for the purposes of 
evaluating the character count, the total of 852 incoming texts was reduced to 819 questions. The average character count for incoming questions was 60.9 , with a slightly lower median of 49 characters. For outgoing responses, the average was about double, at 119.5 characters, and a median of 110 characters. The number of answers that used more than 160 characters was 153 (22\%) (Table 2).

\begin{tabular}{|l|l|l|l|l|}
\hline \multicolumn{5}{|c|}{ Number of Characters per Incoming Message (N=819) } \\
\hline High & Low & Median & Average & No. (\%) of questions using over 160 characters \\
\hline 417 & 1 & 49 & 60.9 & $26(3.2 \%)$ \\
\hline
\end{tabular}

The total number of unique patrons was 326 , of which 74 asked questions in multiple transactions. Of this group of 74 , one patron sent questions in 16 separate transactions, but the median number of transactions was only 3 per patron. Only 6 patrons sent 10 or more texts. We determined the number of days that elapsed between the first and last texts sent by repeat users during the entire span of the service's availability. Approximately half of the patrons who sent questions in multiple transactions used the service only across 2 months, and 12 patrons used the service over more than one year (Table 3).

\begin{tabular}{|l|l|l|l|l|}
\hline \multicolumn{2}{|l|}{ TABLE 3 } \\
\hline & Patrons with Multiple Text Transactions (N=74) \\
\hline & Average & Median & High & Low \\
\hline Number of incoming texts per patron & 4.3 & 3 & 16 & 2 \\
\hline Number of transactions per patron & 3.5 & 3 & 16 & 2 \\
\hline Days elapsed between first and last texts & 161.5 & 56 & 1131 & 0 \\
\hline
\end{tabular}

Among these 74 multiple users, we assessed the category of the questions asked by each patron-for example, which patrons asked only directional, only reference, or a mix of both types of inquiries.

- 31 of the 74 (41.8\%) asked only directional (including circulation, equipment, and room reservation) questions.

- 22 patrons (29.7\%) asked only reference questions.

- 20 patrons (27.0\%) asked a mix of reference and directional questions. 
We were interested in seeing whether patrons who were repeat users of the service would ask progressively complex questions. However, in examining the data, there was no discernible pattern in the difficulty of the questions sent.

\section{Discussion}

We wanted to know whether or not the nature of the medium would have any effect upon the types of questions asked. Though we did notice that questions tended to be short, at approximately 61 characters on average, the types of questions patrons asked were very similar to questions asked through chat and face-to-face (Figure 5). A little more than a third of questions were of the "reference" variety, which is a similar percentage to our other virtual reference mediums as well as to the percentage of reference questions asked in-person at our Service Desk (Figure 5).

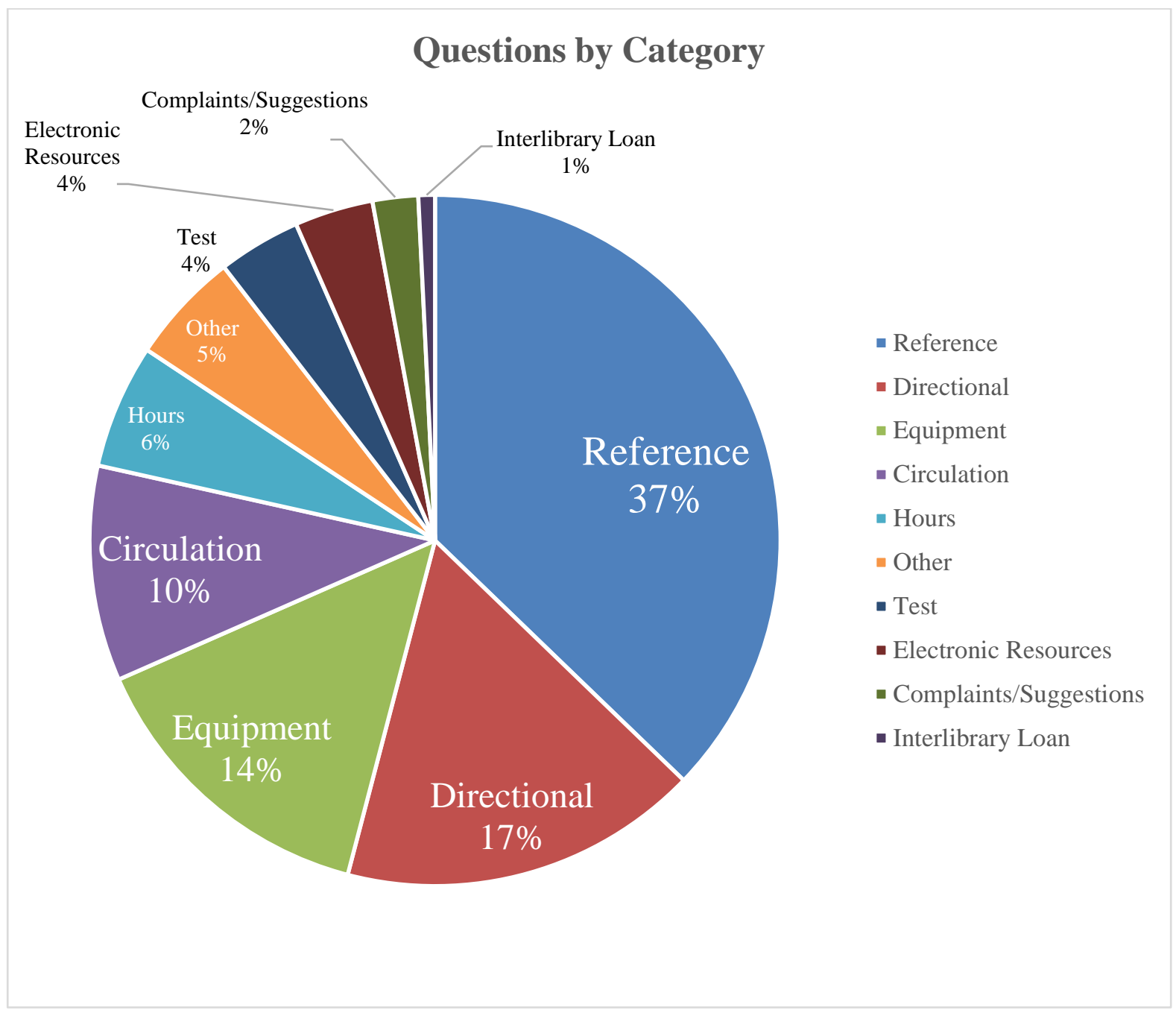

FIGURE 5 Descriptive Category for Questions Received 


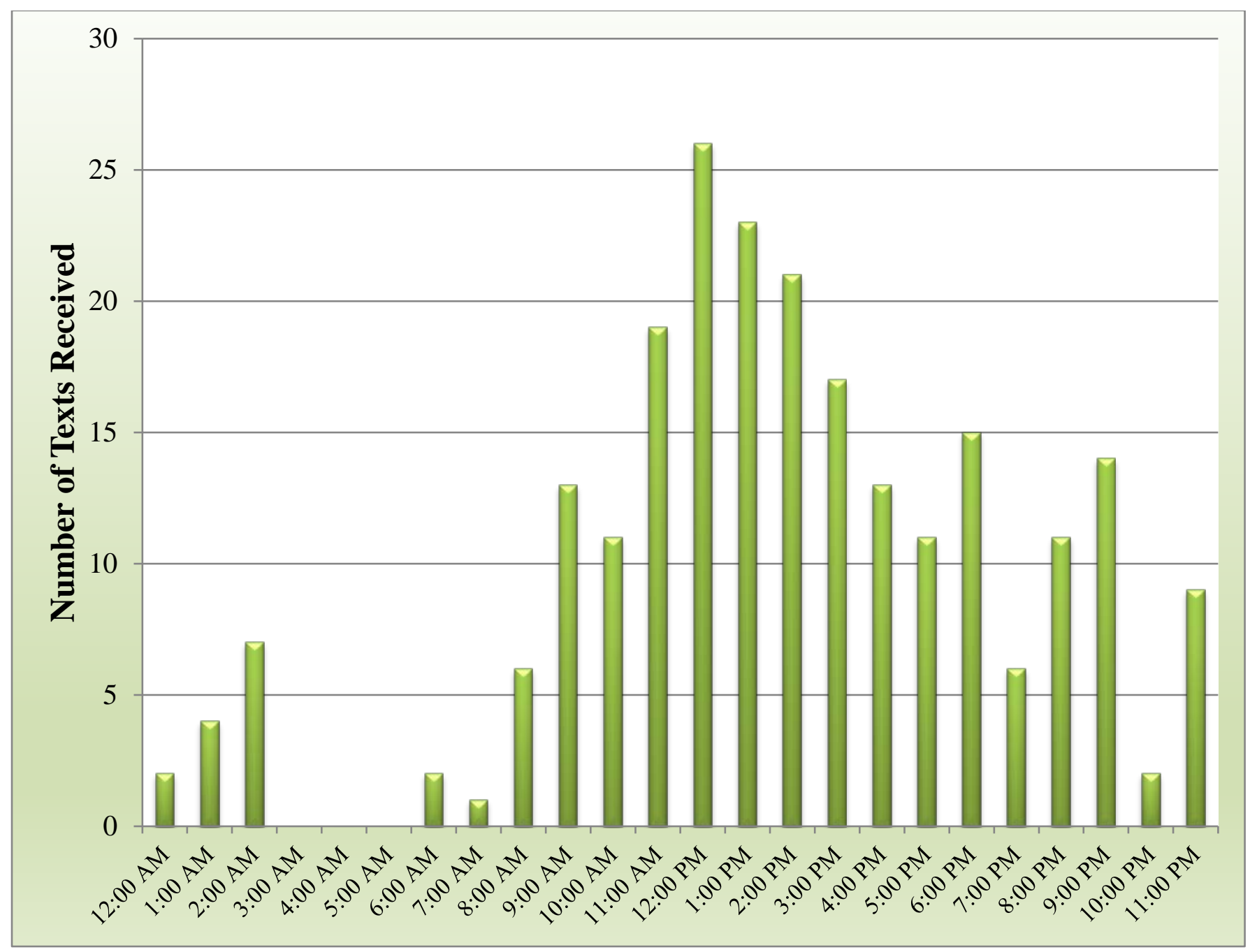

FIGURE 6 Number of Texts Received by Hour of Day, May 2011-March 2012

One of the primary questions we had in implementing text reference as a service was whether it would allow the Libraries to reach patrons we were previously unable to help. Our data suggests this is the case, particularly in tracking when text questions arrive (Figure 2). When we analyzed the first year of usage of Text a Librarian in April 2012, we found that the peak usage time was 12:00 p.m. (Figure 6). Over that year, about $29 \%$ of the texts we received came in the evening hours between 5:00 pm and midnight. However, in analyzing data over the life of the service, the peak time for incoming texts had shifted to $4: 00 \mathrm{pm}$, with $40 \%$ of usage coming in the same 5:00 pm to midnight period (Figure 2). Whereas chat is only available during standard business hours, the service sees continued use (46\%) outside regular business hours. Even so, our general reference librarians were able to respond to the vast 
majority of these questions in a timely fashion. As patrons used the service sparingly, librarians were willing to answer questions after hours. Paradoxically, if the service had been heavily used, they likely would not have been able to keep up with text reference questions received after hours.

In examining how patron use of our virtual reference services evolved over time, we compared the number of incoming questions to our chat, email, and text services during the last five academic years (Figure 7). We observed a dramatic increase in chat usage between 2010 and 2011 (continuing an upward trend from 2009 as well). This most likely resulted from the placement of a chat widget on the home page of our Library website at the beginning of the 2011 academic year. Since 2011, chat use has remained constant. Email transactions also increased dramatically between 2010 and 2011, probably a result of the aforementioned new chat widget that referred patrons to the email service during off hours. However, unlike the chat, email reference numbers steadily increased between 2012 and 2014. Neither chat nor email saw a significant decrease after we implemented text reference-the number of email inquiries decreased less than $5 \%$ in 2012-which leads us to believe that the text service is not cannibalizing users from chat and email services.

Use of text reference declined every year since its introduction. In determining the causes for this decline, and for the service's comparatively low usage numbers overall, we address the correlation with the following factors: low awareness (inconsistent marketing), the cost of texting, and preference for chat services over other virtual reference options. We saw the greatest usage spike in September of 2011, which coincided with the initial marketing push coordinated through the Libraries' Communications \& Marketing department. This included table tents in the library, print handouts, instructions on the "Ask a Librarian" page of the Library website, a news story featured on the Library's home page, as well as a story in the student newspaper, The Daily Toreador. Following the initial campaign, individual librarians have promoted the service during instruction sessions, library tours, resource fairs, and similar events. Since September 2011, use of text reference has fallen and not recovered. The initial marketing effort appears to have had a direct, positive effect on the use of the service at that time. 


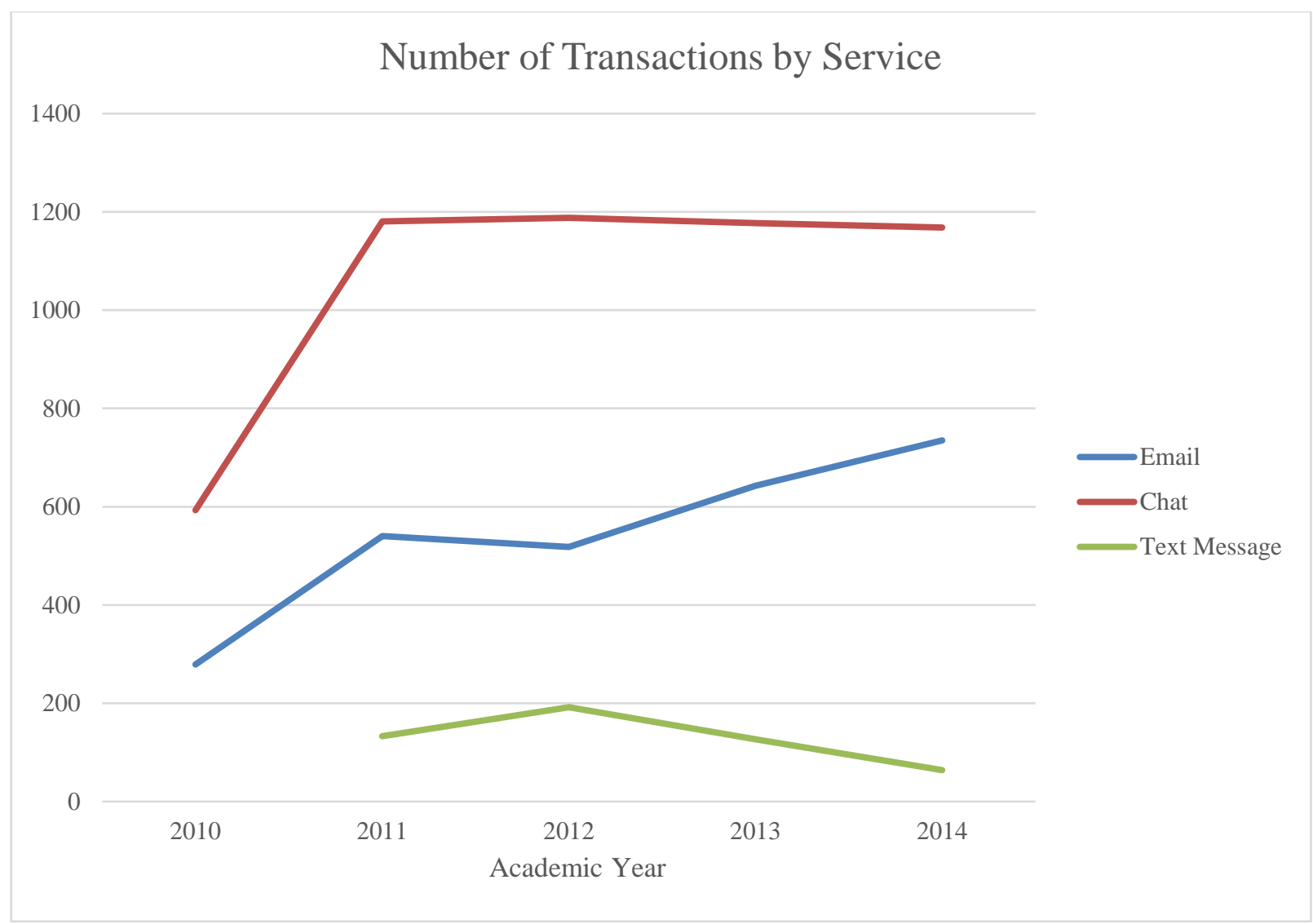

FIGURE 7 Number of Transactions by Service Medium

One of the difficulties in building student awareness and use of Text a Librarian has been the problem of meeting patrons at the point of need. In place of a marketing campaign, we currently rely on librarians to make patrons aware of text reference during their instruction and demonstration sessions. The problem with this model is students might remember that the service exists, but not the necessary steps in sending their first text. While there is detailed information on the Library website, if students look for instructions, it is often easier at that point to use chat or email reference options to fill their immediate need. Ideally the marketing campaign would not have ended, but as new services launched, they took priority over marketing efforts for text reference.

As previous researchers have concluded, it appears unlikely that the cause for underwhelming text reference usage is due to the cost of texting (Pearce et al., 2010). As evidenced by the high number of "thank you" texts received, our library patrons are not restricting themselves to sending the lowest possible number of texts in a conversation. The latest available data from 2010 , indicates approximately $75 \%$ of teenagers had mobile plans with unlimited texting, and only $13 \%$ of teens paid per text message 
sent (Lenhart, Ling, Campbell, \& Purcell, 2010). Several articles discuss a concern among librarians about how they could possibly answer questions in 160 characters (Stahr, 2010; Pearce et al., 2010), with some SMS reference software (including Text a Librarian) even going so far as offering the capability to translate a librarian's reply into texting shorthand (Stahr, 2010). This concern about the character count appears to be overstated. Our outgoing responses averaged 119 characters. The percentage of replies using more than 160 characters, and thus requiring 2 texts, was only $22 \%$.

Another important point of consideration is that any patron with a smartphone could most likely obtain assistance through a chat service as easily as via text reference. Only a few years ago, a majority of mobile phones were not integrated with email accounts or even capable of accessing library websites, let alone opening a flash-based chat service. The technological capabilities and proliferation of mobile devices have advanced rapidly. The 2010 ECAR Study of Undergraduate Students and Information Technology reported that nine out of ten students used text messaging daily, and about half of the students surveyed used mobile devices to access the Internet on a daily basis (Smith \& Caruso, 2010). The 2013 ECAR Study found that more than 75\% percent of students owned smartphones, and it was common for students to own three or four Internet-capable devices (Dahlstrom, Walker, \& Dziuban, 2013). Mobile phones and tablets are rapidly becoming a suitable replacement for laptops and desktop computers. Given the user preference for chat over text reference as indicated in the literature (Chow \& Croxton, 2014; Chow \& Croxton, 2012), and as indicated in this study by higher usage of chat services, it is unlikely that patrons will ever use text reference in greater numbers than other virtual reference services. If the convenience of chatting from a smartphone is comparable to texting, perhaps following Poole's (1985) Principle of Least Effort, users will continue to exercise their preference for chat over text reference.

\section{Limitations and Further Study}

There are a few limitations to our study to consider. First, we had no identifying information on the status of our text reference users - whether they were undergraduates, graduate students, faculty, or not affiliated with the university; thus we were unable to analyze use among our patron populations. Further, the small sample size due to low usage of the service may have skewed the findings. Finally, our 
particular reference model and associated hours of in-person availability may have affected the number and type of virtual reference questions, including text.

We recommend further research to assess which marketing or promotional strategies result in the greatest increase of text reference usage. Additionally, a long-term study to track how the implementation of new and different reference models affects virtual reference services would be valuable. There is also a dearth of reporting on other integrations of library services and text-for example, catalogs/discovery systems that text call numbers or location information, integrated library systems that send overdue notices via text, and event promotions and fundraising requests. Analyzing patron demand and acceptance of these tools would be a further step in assessing the usefulness of text message services for libraries.

Future studies could analyze the impact that increased tablet use has on text reference and on virtual reference in general. Will the growing popularity of tablets cause more users to request research assistance via email and chat (and possibly video chat), abandoning text reference in the process? Other opportunities for future research could assess any impact on text reference services resulting from mobile users' preferences for alternative texting apps, such as WhatsApp, Kik, Viber, or Facebook Messenger, in place of traditional SMS texting.

\section{Conclusion}

Text reference occupies an interesting role in a suite of virtual reference services. On one hand, text functions similarly to chat reference during regular business hours, in that it can provide synchronous assistance. It also operates similarly to email reference, in that questions received outside of business hours can be answered asynchronously. With this in mind, it is important to ask what role text reference serves. Three years of experience with the service leads us to believe it does indeed serve a purpose, though its place is more of a niche than a cornerstone of virtual reference.

Over three years, the service averaged less than 15 text reference transactions per month. Compared with chat and email reference services, it is not a heavily used reference medium. That said, there is evidence suggesting its value. When the service first launched, $29 \%$ of questions arrived between 5:00 p.m. and midnight. Over time, that percentage increased to $40 \%$. Partially because text functions as a synchronous or asynchronous service, it is proving useful beyond standard business hours. 
Our data suggests that the potential costs and the 160 character limitation of the text medium are not a hindrance to the service. If necessary, lengthy messages continued in a second message. Further, patrons often sent multiple messages themselves, and very frequently concluded sessions by sending a "thank you" text. The service received a significant number of reference questions, often requiring multiple texts between patrons and the librarian. When we launched the service, there was a concern that text reference would cannibalize use from chat and email, our other virtual reference mediums. Yet, our data indicates that has not been the case. Use of chat has remained remarkably consistent over the last three years, and use of email reference has actually increased. We attribute low text reference use to the difficulties inherent in the medium and in marketing the service, and to user preference for chat.

Despite the medium's limitations, text reference can play an important role among a suite of virtual reference services. Integration with library systems could also extend the service's utility. To that end, we deployed a support widget on our LibGuides that connects to Mosio's text capabilities and allows patrons to ask a question via the website but receive a response via text message. With greater awareness and integration with library systems, we expect to see an increase in use. However, text reference has already allowed us to serve our patrons at a point of need that chat and email were unable to reach previously. In doing so, we are expanding our ability to help our patrons and providing one more connection to library reference services. 


\section{References}

Breitbach, W., \& Prieto, A. G. (2012). Text reference via Google Voice: A pilot study. Library Review, 61(3), 188-198.

Brooks, M., \& Zubarev, M. (2012). Another lane on the information highway? A case study of experimenting with text message reference. The Reference Librarian, 53(2), 170-181.

Cassidy, E. D., Colmenares, A., \& Martinez, M. (2014). So text me-Maybe: A rubric assessment of librarian behavior in SMS reference services. Reference \& User Services Quarterly, 53(4), 300312.

Chow, A. S., \& Croxton, R. A. (2012). Information-seeking behavior and reference medium preferences. Reference \& User Services Quarterly, 51(3), 246-262.

Chow, A. S., \& Croxton, R. A. (2014). A usability evaluation of academic virtual reference services. College \& Research Libraries, 75(3), 309-361.

Cole, V., \& Krkoska, B. B. (2011). Launching a Text a Librarian service: Cornell's preliminary experiences. The Reference Librarian, 52(1-2), 3-8.

Dahlstrom, E., Walker, J.D., and Dziuban, C. (2013). ECAR Study of Undergraduate Students and Information Technology, 2013. Louisville, CO: EDUCAUSE Center for Analysis and Research.

Herman, S. (2007). SMS reference: Keeping up with your clients. The Electronic Library, 25(4), 401-408.

Hill, J. B., Hill, C. M., \& Sherman, D. (2007). Text messaging in an academic library: Integrating SMS into digital reference. The Reference Librarian, 47(1), 17-29.

Lenhart, A., Ling, R., Campbell, S., \& Purcell, K. (2010). Teens and mobile phones: Text messaging explodes as teens embrace it as the centerpiece of their communication strategies with friends. Washington, DC: Pew Internet \& American Life Project. http://pewinternet.org/Reports/2010/Teens-and-Mobile-Phones.aspx. 
Luo, L. (2012). Professional preparation for "Text a Librarian." Reference \& User Services Quarterly, 52(1), 44-52.

Pearce, A., Collard, S., \& Whatley, K. (2010). SMS reference: Myths, markers, and modalities. Reference Services Review, 38(2), 250-263.

Poole, H. L. (1985). Theories of the middle range. Norwood, N.J: Ablex Pub. Corp.

Ruppel, M., \& Vecchione, A. (2012). "It's research made easier!" SMS and chat reference perceptions. Reference Services Review, 40(3), 423-448.

Smith, S. D. \& Caruso, J. B. (2010). ECAR Study of Undergraduate Students and Information Technology, 2010. Boulder, CO: EDUCAUSE Center for Analysis and Research.

Stahr, B. (2010). Text message reference service: Five years later. The Reference Librarian, 52(1-2), 919. 